\title{
Gelsolin of insect vectors negatively regulates actin-based tubule motility of plant reoviruses
}

\author{
Qian Chen ${ }^{1 \dagger}$, Limin Zheng ${ }^{1,2 \dagger}$, Panpan Zhong ${ }^{1}$, Dongsheng Jia', Yuyan Liu', Qianzhuo Mao and Taiyun Wei ${ }^{1 *}$
}

\begin{abstract}
Most plant reoviruses encode a type of nonstructural protein that assembles tubular structures to package virions for viral spread in planthopper or leafhopper vectors. These tubules are propelled by actin filaments and facilitate viruses to overcome transmission barriers in insect vectors. This is known as actin-based tubule motility (ABTM), in which insect proteins, especially actin-associated proteins participate. To better understand the insect components that play a role in the ABTM, the proteins interacting with tubule protein Pns11 of the Rice gall dwarf virus (RGDV) in the leafhopper vector were investigated. We found that gelsolin, an actin-modulating protein, interacted with Pns 11 in the yeast-two-hybrid system and Sf9 cells. The interaction and co-localization of gelsolin and Pns 11 were also verified in cultured cells and insect bodies of the leafhopper vector. Further, the expression of gelsolin was up-regulated by the RGDV infection both in cultured cells and insects. The knockdown of the gelsolin gene triggered by RNA interference increased viral accumulation, thus increasing the viruliferous rates of the leafhopper vector. This negative association of gelsolin with Pns11 and virus infection revealed that gelsolin negatively affected the ability of the virus to spread by interacting with Pns11 tubules, finally acting to negatively regulate RGDV infection. The results of this study indicate that ABTM is negatively regulated by insects in the coevolution of the insect vector and virus.
\end{abstract}

Keywords: Actin-based tubule motility, Rice gall dwarf virus, Pns11 tubule, Gelsolin, Leafhopper

\section{Background}

Plant reoviruses are transmitted by planthopper or leafhopper vectors in a persistent propagative manner (Attoui et al. 2012). For the persistent propagative transmission, these viruses exploit actin-based tubule motility (ABTM) to overcome the transmission barriers in insect vectors. The tubule refers to the virion-packaging tubule structure that is assembled by a type of virus-encoded non-structural protein via self-interaction. In cultured cells derived from insect vectors, these tubules, propelled by actin filaments in filopodia, protrude from the cellular surface, and penetrate neighboring uninfected cells for persistent viral infection (Wei et al. 2006; Chen et al. 2012; Chen et al. 2013; Jia et al. 2014; Wei and Li 2016). In

\footnotetext{
* Correspondence: weitaiyun@fafu.edu.cn

${ }^{+}$Qian Chen and Limin Zheng contributed equally to this work.

${ }^{1}$ Fujian Province Key Laboratory of Plant Virology, Institute of Plant Virology, State Key Laboratory of Ecological Pest Control for Fujian and Taiwan Crops, Vector-borne Virus Research Center, Fujian Agriculture and Forestry University, Fuzhou, Fujian 350002, People's Republic of China Full list of author information is available at the end of the article
}

insect vectors, virion-packaging tubules propelled by actin filaments pass through multiple tissue and membrane barriers, including microvilli, intercellular junctional complexes, basal lamina, visceral muscle, and plasmalemma of salivary glands (Chen et al. 2012; Jia et al. 2014; Chen and Wei 2016; Wei and Li 2016; Mao et al. 2017). This ABTM represents a specific mode of intercellular viral spread in insect vectors infected by Rice dwarf virus (RDV), Southern rice black-streaked dwarf virus (SRBSDV), or Rice gall dwarf virus (RGDV) (Wei et al. 2006; Chen et al. 2012; Chen et al. 2013; Jia et al. 2014; Wei and Li 2016).

ABTM is mainly conducted by virus-encoded tubular proteins and the actin filament engine. To understand the mechanism of this process, it is essential to investigate the interaction of actin or actin-associated proteins with tubular proteins; it is also necessary to study the regulation mechanism of actin in response to viral infection. Previous studies have shown that the specific interaction of these tubular proteins, including RDV

(c) The Author(s). 2019 Open Access This article is distributed under the terms of the Creative Commons Attribution 4.0 International License (http://creativecommons.org/licenses/by/4.0/), which permits unrestricted use, distribution, and 
Pns10 and SRBSDV P7-1, with the actin of insect vectors contributes to the association of tubules with actin filaments (Wei et al. 2006; Jia et al. 2014). Furthermore, the interaction of RDV Pns10 with insect cytoplasmic actin, which acts as the microvilli backbone of epithelial cells in the leafhopper, likely determines pathogen-vector specificity (Chen et al. 2015a). During the process of ABTM, actin-associated proteins, including myosin, profilin, and cofilin, also positively participate in tubule formation or actin engine regulation (Wei et al. 2008; Liu et al. 2011; Chen et al. 2012; Jia et al. 2014; Mar et al. 2014). In our previous publication, we showed that tropomodulin (Tmod), an actin-associated protein controlling the length and dynamics of the actin-tropomyosin filament, positively regulates RDV Pns10 tubule trafficking via the direct interaction with Pns10 (Chen et al. 2017). This interaction promotes viral infection (Chen et al. 2017). It is believed that the successful operation of ABTM for persistent viral infection is due to the evolutionary arms race and balance between positive and negative regulators.

Gelsolin is a group of actin-binding proteins that are thought to be expressed in all eukaryotes (Kwiatkowski et al. 1986). This protein contains at least 1 repeat with 120 amino acids, and usually possesses 3 or 6 tandem repeats (Kwiatkowski et al. 1986; Garcia-Exposito et al. 2013). This approximately $80-\mathrm{kDa}$ protein severs the long actin filaments to promote the reorganization of the actin skeleton, causes morphological changes to the cytomembrane, and ultimately leads to the formation of filopodia and lamellipodia (Lu et al. 1997; Safiejko-Mroczka and Bell 2001; Mazur et al. 2010; Garcia-Exposito et al. 2013). Gelsolin also engages in viral infection and pathology, playing different roles depending on the virus. In the process of Human immunodeficiency virus type 1 (HIV-1) infection, gelsolin acts as a barrier that limits viral infection in the prefusion step to control the efficiency of early viral infection (Garcia-Exposito et al. 2013). Gelsolin can also regulate the transportation of Minute virus of mice (MVM) from the nucleus to the cell periphery and release the virus into the culture medium (Bar et al. 2008). In vivo, gelsolin is found to be entirely repressed in patients suffering from the Hepatitis B virus (HBV), thus it is proposed as a potential biomarker in HBV-related liver pathologies (Marrocco et al. 2010). However, it is unknown whether gelsolin plays a role in viral pathogens infection in insects.

RGDV, a member of the genus Phytoreovirus in the family Reoviridae, contains 12 double-stranded RNA (dsRNA) segments (S1-S12) encoding six structural proteins (P1, P2, P3, P5, P6, and P8) and six nonstructural proteins (Pns4, Pns7, Pns9, Pns10, Pns11, and Pns12) (Moriyasu et al. 2000; Moriyasu et al. 2007; Zhang et al. 2008). This virus is transmitted by the leafhopper vectors
Recilia dorsalis and Nephotettix cincticeps (Hemiptera: Cicadellidae) in a persistent propagative manner. In the leafhopper vectors, RGDV initially infects the epithelium of the filter chamber and launches viral replication. The newly formed Pns11 proteins assemble into tubular structures for the packaging of progeny virions (Chen et al. 2013). Propelled by the ABTM, these virion-containing tubules cross the basal lamina into the visceral muscles, from where RGDV spreads throughout the entire midgut and hindgut (Zheng et al. 2015). In the later stage of the viral infection, RGDV releases from salivary glands. The virion-containing structures formed by Pns11 serve as a vehicle to pass through actin-based plasmalemma into vector salivary gland cavities, finally overcoming the last membrane barrier for viral transmission (Mao et al. 2017). For RGDV transovarial transmission, virion-containing Pns11 tubules cross through actin-based junctions between follicular cells of the ovary or through actin-based microvilli of follicular cells and penetrate the oocyte of the leafhopper, thus overcoming the transovarial transmission barriers (Liao et al. 2017). However, the mechanism underlying and regulating the RGDV ABTM, in which actin-associated cellular components of insect vectors participate, are still unknown.

In the present study, we investigated the molecular mechanisms for ABTM of RGDV, by combining the yeast two-hybrid assay ( $\mathrm{Y} 2 \mathrm{H})$, baculovirus expression system, immunofluorescence assay, and RNA interference (RNAi) technique based on the cultured cells and insect bodies. We determined that gelsolin, an actin-modulating protein of the insect vector, negatively regulated the ABTM of RGDV by directly interacting with the tubular protein Pns11, ultimately negatively affecting the ability of the virus spread to infect the insect vector.

\section{Results}

\section{Pns11 interacts with gelsolin in vitro}

Because the Pns11 tubule is transmembrane and highly hydrophobic, a membrane protein-specific $\mathrm{Y} 2 \mathrm{H}$ system, the DUALmembrane system, was utilized to screen the proteins of $N$. cincticeps interacting with RGDV Pns11. A cDNA library was constructed using the adults of $N$. cincticeps as the source of mRNA for the $\mathrm{Y} 2 \mathrm{H}$ assay, and the Pns11 gene was cloned into the pBT3-STE to generate the bait pBT-STE-Pns11. Among the positive colonies picked from this library screening, gelsolin showed a high screening frequency, thus was utilized for further analyses. Based on the transcriptome data from N. cincticeps in our lab, the open reading frame (ORF) of gelsolin was amplified. The coding region of the ORF was $2277 \mathrm{bp}$, containing 6 repeats of the homologous gelsolin domains of the superfamily (Fig. 1a). The ORF of gelsolin additionally shared significant sequence similarities with the gelsolin sequences from other insect species. 


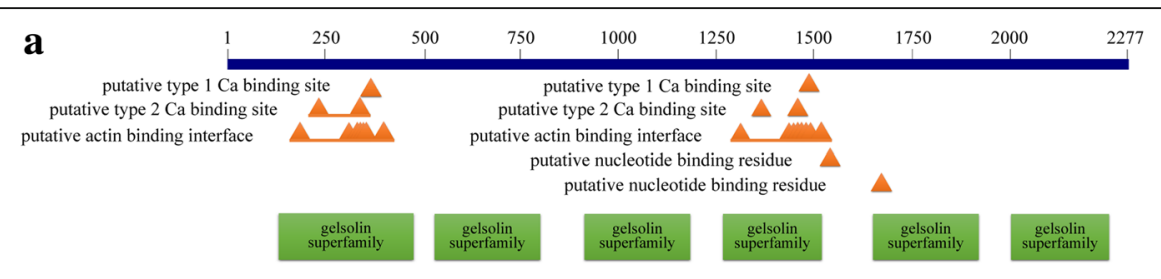

b

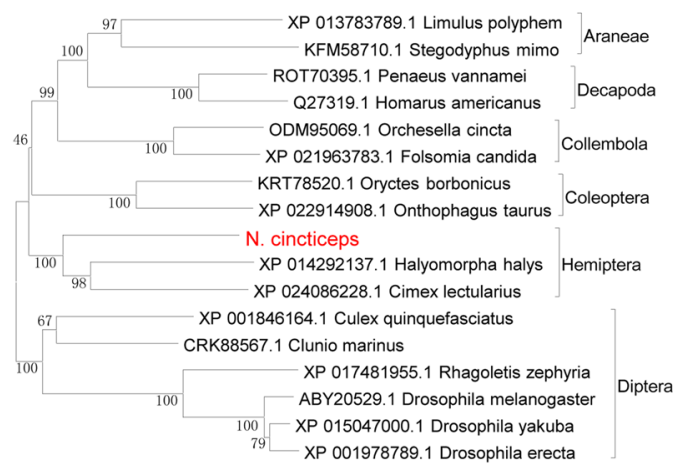

c

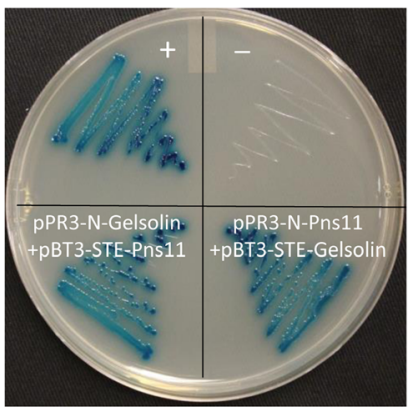

$\overrightarrow{0.05}$

d

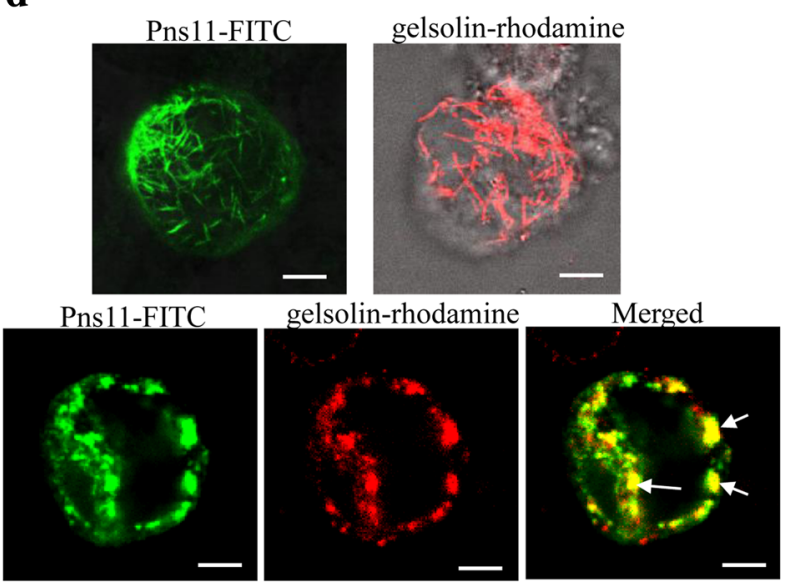

Fig. 1 Interaction of Pns11 with gelsolin in $\mathrm{Y} 2 \mathrm{H}$ and Sf9 systems. a Schematic representation of the gelsolin gene with the domain of the gelsolin superfamily and other binding sites. $\mathbf{b}$ Phylogenetic relationships of gelsolin orthologs of $N$. cincticeps with counterparts. The available sequences were aligned using Clustal W, and phylogenetic trees were reconstructed using the neighbor-joining analysis with $P$-distance using MEGA 5.0. Reliability of the phylogenetic trees was estimated by calculating bootstrap confidence limits based on 1000 replicates. $\mathbf{c} Y 2 \mathrm{H}$ assay of Pns11-gelsolin interaction. Transformants on plate of SD/-Leu-Trp-His-Ade medium were as follows: +, positive control, i.e., pTSU2-APP/pNubGFe65; -, negative control, i.e., pTSU2-APP/pPR3-N. d Co-localization of Pns11 and gelsolin in baculovirus expression system. Sf9 cells infected with recombinant baculoviruses encoding Pns11 or gelsolin were fixed at 48 hpi and prepared for immunofluorescence microscopy. Cells were immunostained with Pns11-FITC for Pns11, or the monoclonal antibody against strep tag and the secondary antibody of anti-mouse lgG-rhodamine for gelsolin. White arrows indicate the co-localization of Pns11 and gelsolin. Bars, $10 \mu \mathrm{m}$

The predicted protein products (759 amino acid residues) possessed characteristic gelsolin domains (Pfam 00626) including actin binding interface and calcium binding sites (Fig. 1a). The phylogenic analysis revealed that the amino acid sequence of gelsolin clustered with the gelsolin sequences of other insect species in the order Hemiptera (Fig. 1b). In the further $\mathrm{Y} 2 \mathrm{H}$ analysis, the protein expressed by the full-length
cDNA of gelsolin showed a strong interaction with Pns11 (Fig. 1c).

To confirm this interaction, we next used the Bac-to-Bac Baculovirus Expression System to overexpress Pns11 or gelsolin in Sf9 cells. Sf9 cells were inoculated with recombinant baculovirus encoding Pns11 or gelsolin fused with a strep-tag. At $48 \mathrm{~h}$ post inoculation (hpi), cells were fixed and immunostained with Pns11-specific IgG 
conjugated to fluorescein-5-isothiocyanate (Pns11-FITC) for Pns11 or the monoclonal antibody against strep tag and the secondary antibody of anti-mouse IgG-rhodamine for gelsolin. As observed by immunofluorescence, Pns11 expressed in the tubule structures within the cytoplasm (Fig. 1d), as previously described (Chen et al. 2013). Gelsolin expressed in filament-like structures (Fig. 1d). Then Sf9 cells were co-infected with recombinant baculoviruses encoding Pns11 or gelsolin. In the overexpressed Sf9 cells, gelsolin co-localized with Pns11 in an inclusion-like structure instead of a filament-like structure in gelsolin single-expressed cells or tubule structures in Pns11 single-expressed cells. Together with the results from $\mathrm{Y} 2 \mathrm{H}$ analysis, these data suggest that Pns11 interacted with gelsolin in heterologous expression systems (Fig. 1d).

\section{Co-localization of gelsolin and Pns11 in N. cincticeps}

To validate this interaction in vivo, antibodies against gelsolin for the co-localization assay were prepared. We constructed a plasmid of gelsolin fused with $6 \times$ His-tag at the $\mathrm{N}$-terminus and expressed the fusion protein His-gelsolin. The mouse polyclonal antibodies against His-gelsolin were prepared and tested for specificity using a western blot assay. As expected, gelsolin was detected as an $85-\mathrm{kDa}$ protein in extracts from virus-infected cultured cells and insect vectors, but not in the PBS control (Fig. 2a), verifying the specificity of antibodies against gelsolin.

Then, immunofluorescence assays were performed to detect the expression and location of gelsolin in cells. At 44 hpi, RGDV-infected cultured cells were treated with Pns11-FITC and gelsolin-specific IgG conjugated to rhodamine (gelsolin-rhodamine). The results showed that in mock-infected cells, gelsolin was detectable as the thread-like structures (Fig. 2b). In viral infected cells, Pns11 expressed in numerous tubule structures protruding from the cell surface (Fig. 2b), which was similar to previously published results (Chen et al. 2013). Double immunolabeling with Pns11-FITC and gelsolin-rhodamine revealed that the gelsolin co-localized with Pns11 tubules protruding from or scattering outside cells (Fig. 2c). It is possible that the alternation of the expression pattern of gelsolin was due to the direct interaction of gelsolin with Pns11.

We further examined the co-localization of gelsolin and Pns11 in viruliferous $N$. cincticeps. In non-viruliferous $N$. cincticeps, gelsolin localized in visceral muscles, including longitudinal and circular muscle fibers that surrounded the midgut (Fig. 3a). In viruliferous $N$. cincticeps, abundant Pns11 tubules were associated with circular muscle fiber bundles connected to single longitudinal muscle fibers at 4 days post-first access to diseased plants (padp) (Fig. 3a). Double immunolabeling with Pns11-rhodamine and gelsolin-FITC indicated that Pns11 tubules located in gelsolin-labeled visceral muscles (Fig. 3b). Thus, these co-localization assays verified that Pns11 tubule interacted with gelsolin in vivo.

\section{Gelsolin negatively regulates virus accumulation}

To understand the functional role of gelsolin in viral infection, the expression of gelsolin in RGDV-infected cultured cells was analyzed. The RT-qPCR assay showed that the relative expression of gelsolin was up-regulated by RGDV infection at $48 \mathrm{hpi}$ (Fig. 4a-I). A western blot assay also demonstrated the high accumulation of gelsolin in RGDV-infected cultured cells at $48 \mathrm{hpi}$, in contrast to that of uninfected cells (Fig. 4a-II).

RNAi was conducted to investigate the effect of reduced gelsolin expression on viral infection in the leafhopper vector. The cultured leafhopper cells were treated with dsRNAs specific for gelsolin (dsgelsolin) or green fluorescent protein (dsGFP) via Cellfectin-based transfection. The viability tests showed the absence of toxicity to cultured cells caused by dsRNAs (data not shown). The relative expression of gelsolin, Pns11, and P8 at 44 hpi was analyzed using RT-qPCR. A notable reduction in the relative gelsolin expression was observed in the dsgelsolin-treated cultured cells, compared to dsGFP treatment (Fig. 4b), indicating that the expression of gelsolin was knocked down via the transfection with dsgelsolin. More importantly, the relative expression of Pns11 and P8 in the dsgelsolin treatment was higher than that of the dsGFP treatment (Fig. 4b), suggesting that the expression of gelsolin was negatively associated with Pns11 expression and viral accumulation. The immunofluorescence analysis of 25 pictures for each treatment showed that the dsgelsolin treatment caused an increase in the viral infection rate and an increase in the expression of Pns11 tubules from approximately $40 \%$ to $80 \%$, compared with dsGFP-treated cells at 44 hpi (Fig. 4c). These results revealed that the knockdown of gelsolin improved the expression of Pns11, finally increasing the RGDV accumulation in cultured cells.

We also analyzed the viral titer in $N$. cincticeps vectors with knocked down gelsolin. Non-viruliferous second instar nymphs were allowed a 3-day acquisition on RGDV-infected rice plants, then microinjected with dsRNAs. At 6 days padp, the mean viral genome copies of leafhoppers microinjected with dsgelsolin $\left(2.11 \times 10^{8}\right.$ copies/ $\mu \mathrm{g}$ insect RNA) was significantly higher than that of the dsGFP treatment $\left(1.10 \times 10^{8}\right.$ copies/ $\mu \mathrm{g}$ insect RNA), which was consistent with the results from the cultured cells (Fig. 4d). The increase in the viruliferous rate by more than $50 \%$, caused by the knockdown of gelsolin, was also detected at 4 days padp (Fig. 4e). Because gelsolin was an important protein participating in the architecture of the cytoskeleton, a survival assay was performed to examine the effects of knocking down gelsolin on non-viruliferous insect survival. Our test 


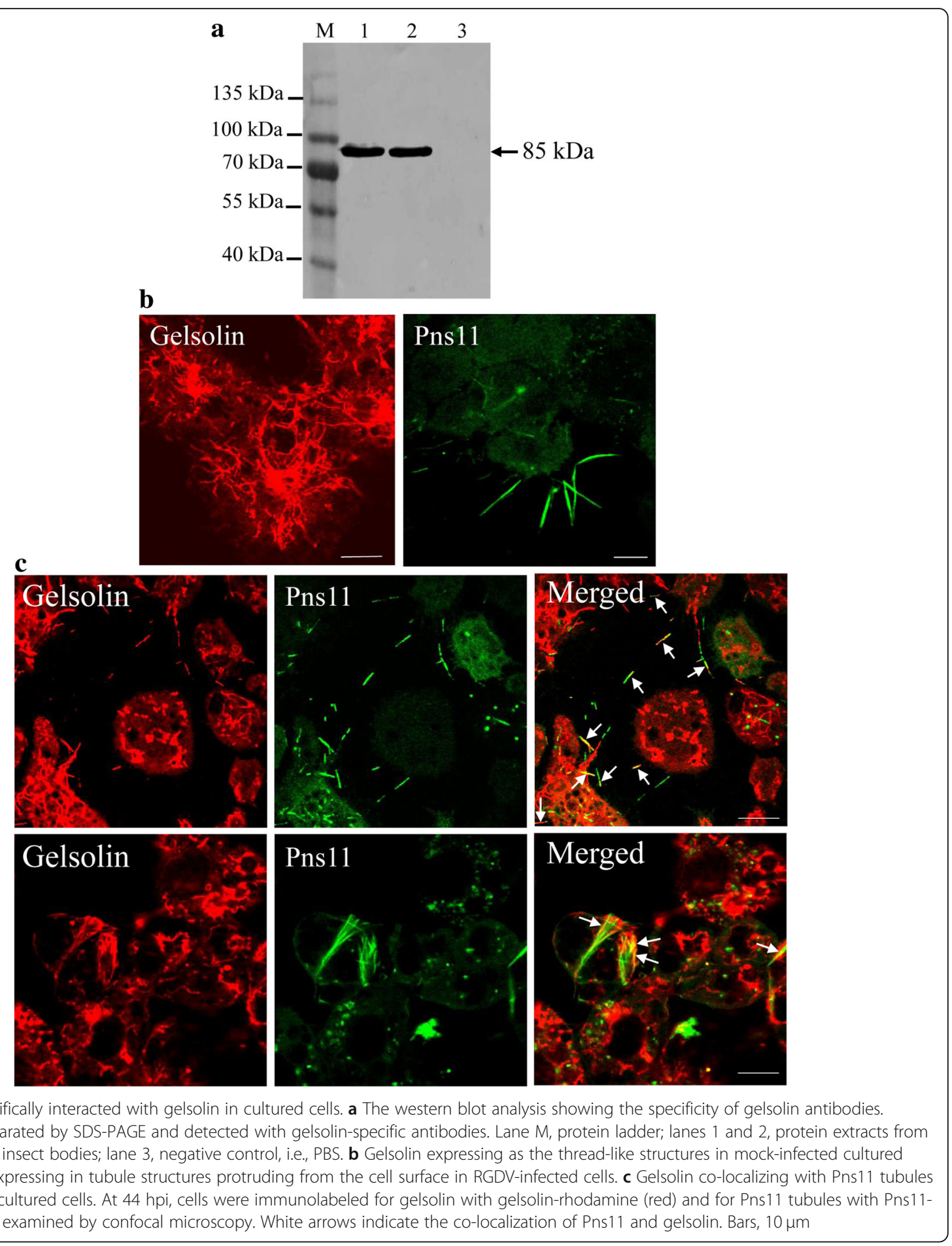

showed the lack of significant difference of survival rate between dsGFP and dsgelsolin treatment (Additional file 1: Figure S1). Thus, the knockdown of gelsolin did not affect the normal survival of leafhoppers (Additional file 1: Figure S1).

Overall, the results showed that knocking down the gelsolin gene increased viral accumulation and relative gene expression of Pns11 in cultured cells and insect bodies, resulting in the increase in the viruliferous rate of leafhopper vectors. These results are consistent with the result from the Sf9 cells, indicating that co-overexpression of Pns11 and gelsolin inhibited the expression pattern of each protein and formed an inclusion structure. Thus, gelsolin played a negative role in the regulation of Pns11 


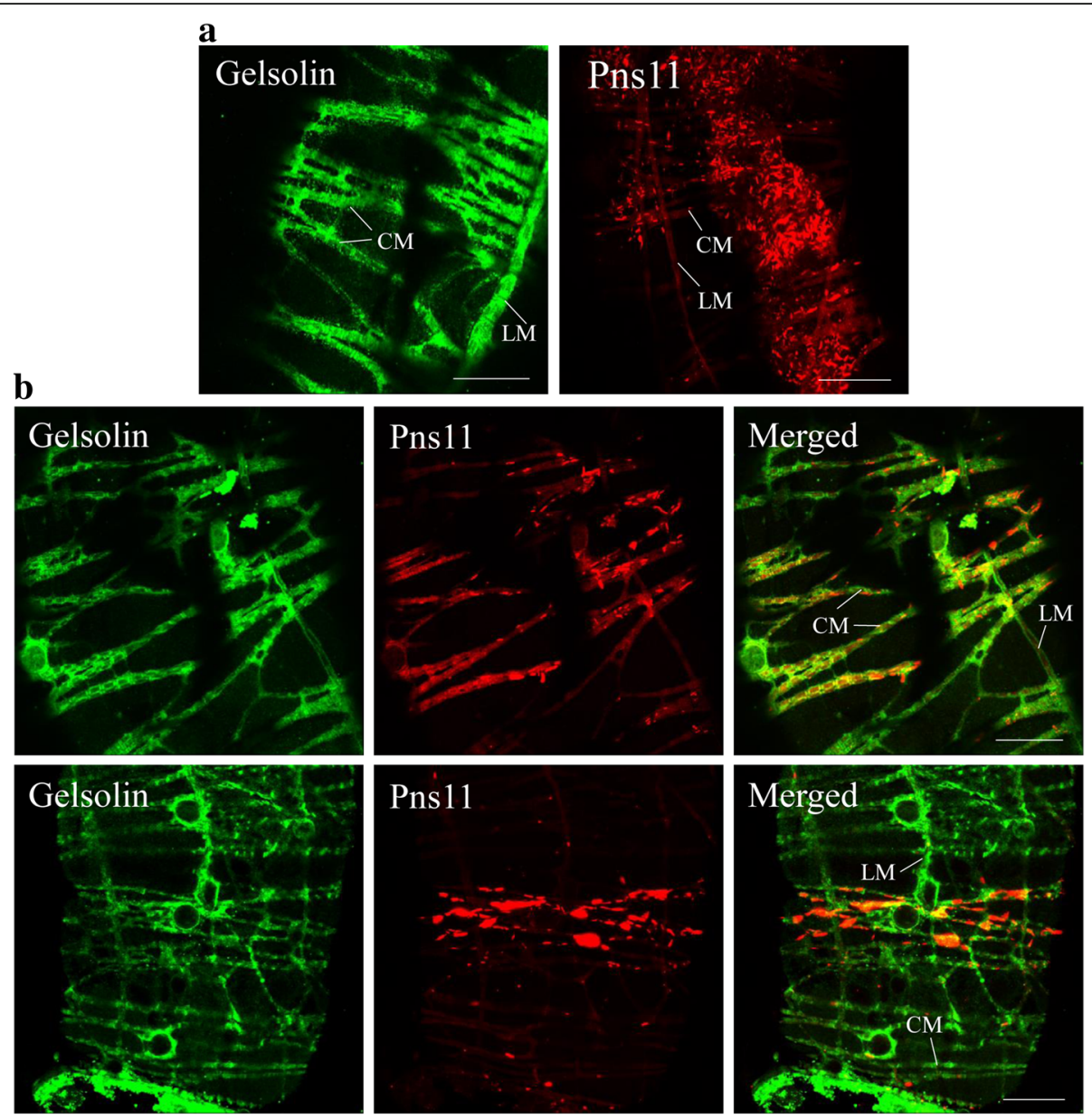

Fig. 3 The co-localization of Pns11 with gelsolin in viruliferous insect vector. a Gelsolin and Pns11 tubules localized in the visceral muscles of the gut in the nonviruliferous and viruliferous insects, respectively. $\mathbf{b}$ The co-localization of Pns11 tubules with gelsolin on the visceral muscles of the gut in viruliferous insects at 6 days padp. Leafhopper organs were immunolabeled for Pns11 tubules with Pns11-rhodamine (red) and for gelsolin with gelsolin-FITC (green), then examined by confocal microscopy. CM, circular muscle. LM, longitudinal muscle. Bars, $20 \mu \mathrm{m}$

expression by direct interaction, ultimately indirectly inhibiting the RGDV infection in insect vectors.

\section{Discussion}

The investigation on the regulators of ABTM is beneficial for the improved understanding of the mechanism of ABTM. Our previous study revealed that Tmod, a positive regulator of RDV ABTM, functioned in viral infection by directly interacting with tubule protein Pns10 (Chen et al. 2017). Because Tmod could control the length and stability of the actin-tropomyosin filament, Pns10 utilizes Tmod to elongate the Pns10 tubules to accomplish ABTM (Chen et al. 2017). In the present study, gelsolin was identified as a negative regulator of the RGDV ABTM. We found that the gelsolin of $N$. cincticeps possessed the general characteristics of the gelsolin superfamily, including the repeats and the molecular weight of the protein (Fig. 1 and Fig. 2). Combined with the transcriptome data from $N$. cincticeps and the results of the phylogenetic analysis, we confirmed the reliability of the gelsolin sequence. Furthermore, this study demonstrated that in both the cultured cells and insect bodies of the leafhopper vector, the expression of gelsolin was up-regulated with RGDV infection. Gelsolin negatively engaged in RGDV infection by interacting with the Pns11 virus-encoded tubular protein (Fig. 1, Fig. 2, Fig. 3 and Fig. 4). In the Sf9 system, the overexpression of gelsolin inhibited the formation of Pns11 tubules (Fig. 1), indicating that gelsolin negatively impacted typical tubule assembly.

To determine the mechanism by which gelsolin played a negative role in RGDV ABTM, we analyzed the process of dynamic actin elongation based on previously published data. The growth or shrinkage of actin filaments is accomplished by the assembly or disassembly of actin subunits from both ends of the filament (Cooper and Schafer 2000). The two ends of the polarized actin filament, barbed (plus or fast-growing) and pointed (minus 
a

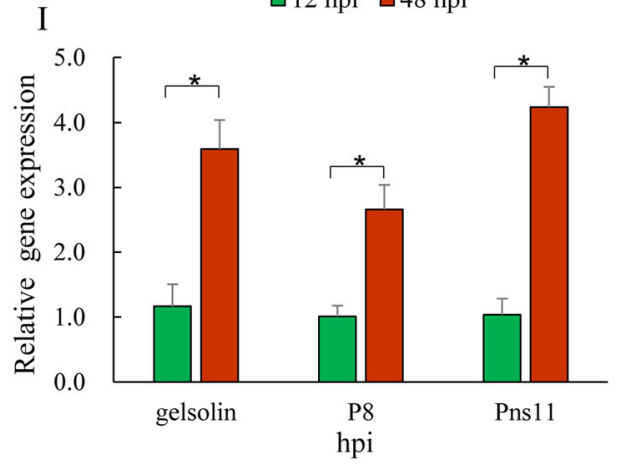

b

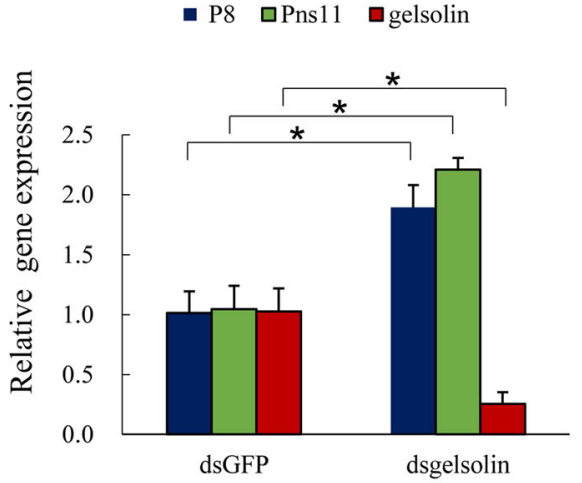

c
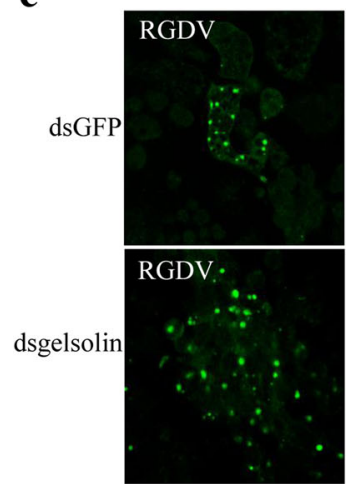

\section{Pns11}
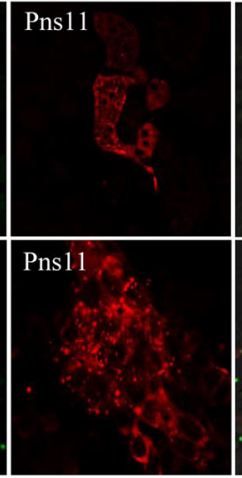

II

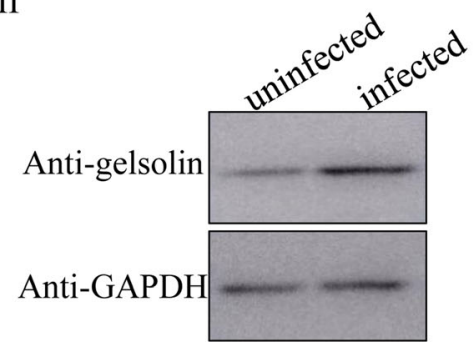

d

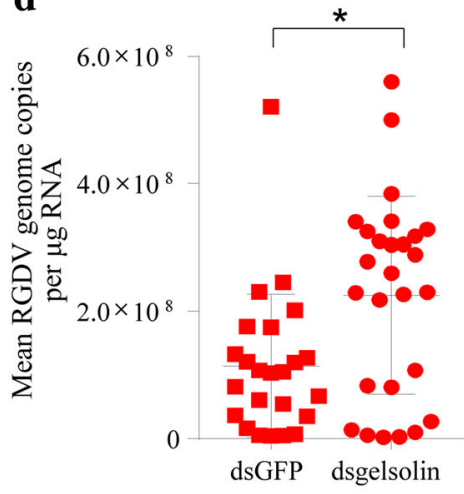

$\mathbf{e}$

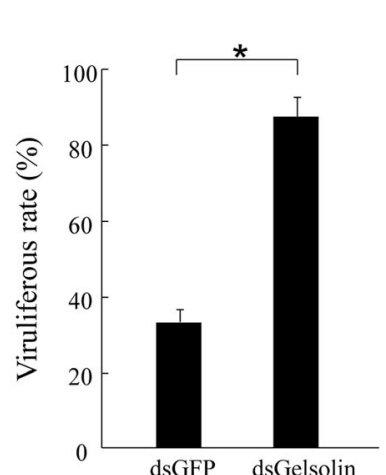

Fig. 4 Gelsolin negatively regulated Pns11 expression and RGDV accumulation. a RGDV infection up-regulated the expression of gelsolin. (I) RTQPCR assay showing the relative expression of gelsolin, Pns 11, and outer capsid protein P8 in cultured cells at 48 hpi, compared to that at 12 hpi. The expression levels of P8, Pns11, and gelsolin in RGDV-infected cells at $12 \mathrm{hpi}$ were normalized as 1. Error bars indicate standard deviations from three independent RT-qPCRs. (II) Western blots assay showing the expression of gelsolin in uninfected and RGDV-infected cultured cells at 48 hpi. b Knockdown of gelsolin improved the level of Pns11 expression and RGDV accumulation in cultured cells. The expression levels of P8, Pns11, and gelsolin in dsGFP-treated cells were normalized as 1. Error bars indicate standard deviations from three independent RT-qPCRs. A onetailed t-test was conducted in GraphPad Prism 6. The mean results \pm the standard deviations are shown. ${ }^{*} P<0.05$. c Knocking down gelsolin expanded the viral infection area and increased Pns11 expression in cultured cells. Cells were immunolabeled, for virus with RGDV -FITC (green) and for Pns11 tubules with Pns11-rhodamine (red), then examined by confocal microscopy. Bars, $10 \mu \mathrm{m}$. d Knocking down gelsolin increased viral titer in insects. Nonviruliferous second-instar nymphs sequentially were allowed a 3-day acquisition of RGDV and subjected to dsRNAs microinjection, then more than 30 living insects were examined at 6 days padp. e Knockdown of gelsolin increased the viruliferous rate of $N$. cincticeps. The dsRNA-microinjected insects, which then fed on RGDV-infected plant for 2 days, were analyzed for the viruliferous rates by RT-PCR at 4-day padp. Error bars indicate standard deviations from three independent RT-qPCRs. Two-tailed t-test analysis was conducted in GraphPad Prism 6. The mean results \pm the standard deviations are shown. ${ }^{*} P<0.05$ 
or slow-growing), are able to add and lose subunits (Coluccio 1994; Mudry et al. 2003). Tmod preferentially binds to the pointed ends of actin filaments to form a cap, while gelsolin caps the barbed ends of actin filaments for depolymerization (Coluccio 1994; Peddada et al. 2013). To explain the possible process of how gelsolin negatively regulated Pns11 tubules and viral infection, we proposed a model shown in Fig. 5. In nonviruliferous leafhopper vectors, the growth of actin filaments was positively regulated by Tmod via actin polymerization and negatively regulated by gelsolin via actin depolymerization (Fig. 5a). Once insects were infected by RGDV, longer and more actin filaments were acquired for ABTM to accomplish persistent viral infection, resulting in the upregulated expression of actin filament regulators, including gelsolin. Thus, in the viruliferous leafhopper vector, gelsolin depolymerized actin, in order to maintain the normal function of actin filaments. Gelsolin also interacted with Pns11 to block the ABTM (Fig. 5b). Typical tubule formation was inhibited or virion-containing tubules were disassembled when Pns11 interacted with gelsolin (Fig. 5b). When the gelsolin gene was knocked down, more Pns11 tubules were produced and inhibited tubules were reassembled, leading to the recovery of ABTM, ultimately promoting viral infection (Fig. 5c).

From an evolutionary standpoint, successful virus infection can be attributed to the balance achieved by the coevolution of the virus and the insect vector (Dietzgen et al. 2016; Wei and Li 2016). During the process of infection, the virus exploits various resources in the insect, including insect proteins, cellular machinery, and metabolic or immunity pathways for viral propagation and expansion (Wei et al. 2011; Wang et al. 2012; Tang et al. 2014; Liu et al. 2015). For example, Rice stripe virus (RSV) binds cuticular proteins of the planthopper vector

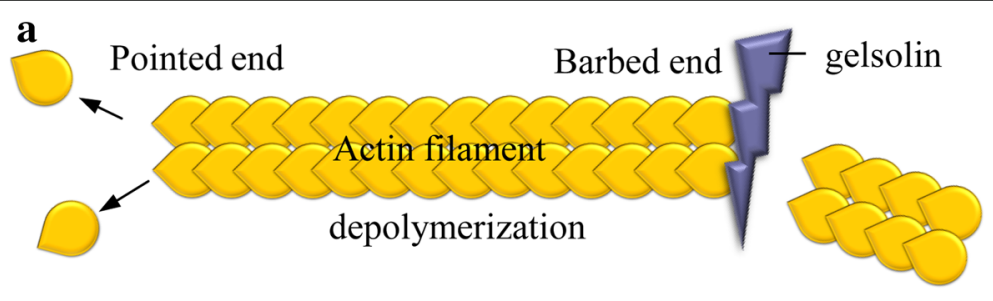

b

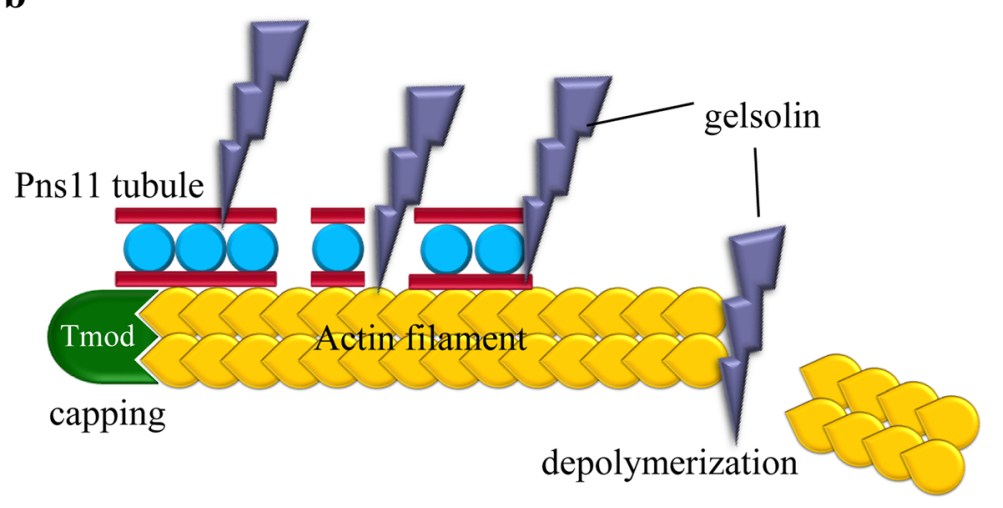

c

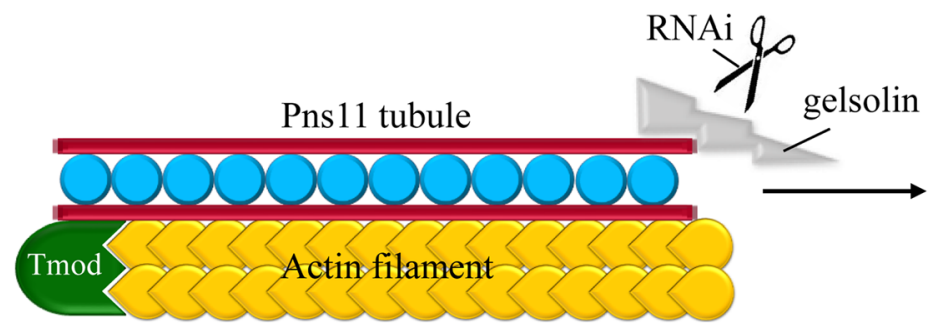

Fig. 5 A proposed model for the ABTM in which gelsolin participates. a The shrinkage of actin filament caused by the depolymerization of actin subunit. The actin filament could be severed by gelsolin at the bared ends, and actin subunits could dissociate from pointed ends. $\mathbf{b}$ In the RGDV-infected cells, the up-regulated gelsolin interacted with Pns11 to disassemble the tubules or inhibit tubular formation, ultimately blocking ABTM. Tmod, a capping protein and positive regulator of ABTM, protected the actin filaments from dissociation at the pointed end. c Knockdown of gelsolin allowed for the reassembly of Pns11 and new tubules assembled, leading to the recovery and promotion of ABTM 
to stabilize viral concentration in the hemolymph (Liu et al. 2015). Dengue virus usurps Rab18 protein, a member of the Rab GTPase family, to facilitate its own replication in mosquito cells (Tang et al. 2014). To control viral infection and limit viral pathology, factors within the insect also regulate viral infection including via the RNAi antiviral pathway and innate immunity responses (Vaidyanathan and Scott 2006; Xi et al. 2008; Cheng et al. 2016; Lan et al. 2016). In the present study, we found a novel negative regulator of viral infection, gelsolin, which modulated viral spread, ultimately controlling the viral infection within the insect vector. This provides insight intonegative regulators of viral infection.

\section{Conclusions}

In the present study, we broadened our knowledge of regulators of ABTM. Gelsolin, a negative regulator of viral infection, was identified and characterized. The gelsolin gene sequence of $N$. cincticeps possessed the general characteristics of the gelsolin superfamily. Gelsolin of $N$. cincticeps interacted with Pns11 in the $\mathrm{Y} 2 \mathrm{H}$ system and baculovirus expression system. The colocalization of gelsolin and Pns11 was confirmed in the cultured cells and intestines of leafhoppers. Furthermore, RGDV infection could significantly up-regulate the gene and protein expression of gelsolin. The knockdown of gelsolin increased the viral infection in cultured cells, the viral titers in leafhopper vectors, and the viruliferous rate of insects. This negative effect of gelsolin on viral infection and Pns11 expression was also consistent with the results of overexpression of Pns11 and gelsolin within the same Sf9 cells. Overexpression of gelsolin in Sf9 cells both colocalized with Pns11 and inhibited the formation of typical Pns11 tubules, suggesting that gelsolin negatively impacted tubular assembly.

Here, we illustrated the existence of a negative regulator of ABTM. This important balance ensured the successful performance of ABTM and protected the insect vector from negative impacts of the virus, ultimately guaranteeing the viral infection in insect bodies. It is believed that further studies will identify additional negative regulators of ABTM.

\section{Methods}

\section{Insects, virus, and cells}

The diseased rice plants were collected from rice fields in Guangdong Province, China and maintained at $25 \pm 3$ ${ }^{\circ} \mathrm{C}$ in a greenhouse. The non-viruliferous $N$. cincticeps leafhoppers were collected from the field in Fujian Province, China and propagated for several generations in cages. Diseased rice plants were propagated via transmission by $N$. cincticeps under greenhouse conditions. The $N$. cincticeps cell line was originally established from embryonic fragments of $N$. cincticeps. The established continuous cultured cells were maintained and passaged for several generations at $25^{\circ} \mathrm{C}$ in Kimura's insect medium as described previously (Kimura and Omura 1988).

\section{$\mathrm{Y} 2 \mathrm{H}$ assay}

Because tubular proteins of plant reoviruses, including Pns11 of RGDV, possess transmembrane domains (Wei et al. 2006; Liu et al. 2011; Chen et al. 2013; Jia et al. 2014), Y2H screening was performed using the DUALmembrane system (Dualsystems Biotech), which is useful for the investigation and identification of the interaction of transmembrane proteins. Full-length cDNA of RGDV Pns11 amplified by PCR was inserted into the bait vector pBT3-STE using the SfiI restriction site, and then was tested for toxicity or self-activation in the yeast strain NMY51. A cDNA library of $N$. cincticeps adults was constructed in the prey vector pPR3-N. Thereafter, the bait and prey were co-transformed in NMY51, and transformants were screened on the SD triple-dropout medium (SD/-Leu-Trp-His) and SD quadruple-dropout medium (SD/-Leu-Trp-His-Ade). Positive clones were selected and streaked on quadruple-dropout/X plates containing X-Gal in a final concentration of $0.8 \mathrm{mg} / \mathrm{mL}$ for color formation in a $\beta$-galactosidase assay. The pTSU2-APP/pNubG-Fe65 interaction served as a positive control, and the pTSU2-APP/pRR3N served as a negative control.

To further analyze the interaction of gelsolin with Pns11, the full-length cDNA of gelsolin was amplified and cloned into the prey vector pPR3-N to construct the plasmid pPR3-N-gelsolin; it was then cloned into the bait vector pBT3-STE to generate the plasmid pBT3STE-gelsolin. The fragment of Pns11 was cloned into the prey vector pPR3-N to construct the plasmid pPR3-N-Pns11. The interaction of Pns11 with gelsolin was investigated further using the following pairings: pPR3-N-gelsolin and pBT-STE-Pns11, pBT3-STE-gelsolin and pPR3-N-Pns11. These prey and bait plasmids were co-transformed in NMY51. The yeast clones were successively cultured on the SD quadruple-dropout medium and then analyzed with the $\beta$-galactosidase assay, as mentioned in the above paragraph.

\section{Antibodies}

Mouse polyclonal antisera against gelsolin were prepared as described previously (Chen et al. 2017). The ORF was amplified without stop codons by RT-PCR. The products were purified and cloned into the Gateway vector pDEST17 (Invitrogen) to fuse with $6 \times$ His-tag at the $\mathrm{N}$-terminus. The resulting plasmid, pDEST17-gelsolin, was then transformed into Escherichia coli strain Rosetta, and the fusion protein His-gelsolin was induced by isopropyl- $\beta$-D-thiogalactopyranoside (IPTG) (Sigma) $(1 \mathrm{mmol} / \mathrm{L})$. Cells were harvested and sonicated, then 
analyzed by SDS-PAGE gel. The protein of the expected size isolated from the gel was used for the preparation of mouse polyclonal antisera against the gelsolin by the GenScript Biotech Corporation, which is approved by the Science Technology Department of Jiangsu Province. IgG was isolated from the specific polyclonal antisera with a protein A-Sepharose affinity column (Thermo Fisher Scientific), and then eluted in phosphate buffered saline (PBS). The specificity of antibodies against gelsolin was tested by western blotting. Total proteins of cultured cells or $N$. cincticeps were extracted and separated using SDS-PAGE. Finally, the total proteins were transferred to a PVDF membrane for detection with the IgG of gelsolin, as described previously (Jia et al. 2012; Chen et al. 2017).

Rabbit polyclonal antisera specific for Pns11 were provided by Dr. Toshihiro Omura from the National Agricultural Research Center, Japan. The purified IgG of Pns11 and gelsolin were conjugated directly to FITC or rhodamine according to the manufacturer's instructions (Thermo Fisher Scientific) to prepare gelsolin-FITC or rhodamine and Pns11-FITC or rhodamine.

\section{Recombinant baculovirus expressing RGDV Pns11 or gelsolin} The infection of Sf9 cells with the recombinant baculovirus vector containing Pns11 of RGDV or gelsolin have been previously described (Chen et al. 2013). In brief, the coding region of the ORF for RGDV Pns11 was amplified, and the coding region of the ORF for gelsolin was amplified by RT-PCR with the reverse primer fused with the sequence of the strep-tag and forward primer. These purified PCR products were individually cloned into the Gateway vector pDEST8 (Thermo Fisher Scientific) to generate a recombinant baculovirus vector containing Pns11 or gelsolin. A recombinant bacmid was generated by introducing the recombinant baculovirus vector into E. coli DH10Bac (Thermo Fisher Scientific). Then, Sf9 cells were inoculated with the purified recombinant bacmid in the presence of Cellfectin II (Thermo Fisher Scientific) according to the manufacturer's instructions. After a high-titer baculoviral stock was generated, Sf9 cells growing on coverslips were infected with recombinant bacmids.

At $48 \mathrm{hpi}$, the Sf9 cells expressing Pns11 or gelsolin fused with the strep-tag were fixed in $4 \%$ paraformaldehyde in PBS for $30 \mathrm{~min}$ and treated with $0.2 \%$ Triton-X for $10 \mathrm{~min}$, as previously described (Chen et al. 2012). Then, the cells expressing Pns11 were incubated with Pns11-FITC antibody. The cells expressing gelsolin were successively incubated with monoclonal antibody against the strep tag (Thermo Fisher Scientific) and the secondary antibody of anti-mouse IgG (whole molecule)-FITC (Sigma). Cells were visualized using a Leica TCS SPE inverted confocal microscope.

\section{Viral infection in cultured cells of leafhopper}

The inoculation of cultured cells with RGDV was initiated as previously described (Wei et al. 2006). Briefly, when confluency of the cultured cells growing on a coverslip (15 mm diameter) reached $80 \%$, the cells were inoculated with purified RGDV at a multiplicity of infection (MOI) of 0.1 or 1 for $2 \mathrm{~h}$, and then washed twice with His-Mg buffer $\left(0.1 \mathrm{M}\right.$ histidine, $0.01 \mathrm{M} \mathrm{MgCl}_{2}, \mathrm{pH}$ 6.2). Finally the cells were covered with growth medium and incubated at $25^{\circ} \mathrm{C}$.

\section{Immunofluorescence of leafhopper cultured cells and dissected organs}

The cultured cells at 44 hpi were fixed in $4 \%$ paraformaldehyde in PBS for $30 \mathrm{~min}$, and then permeabilized in $0.2 \%$ Triton-X for $10 \mathrm{~min}$. The cultured cells were then immunostained with antibodies of gelsolin-rhodamine or Pns11-FITC for $45 \mathrm{~min}$, as described previously (Chen et al. 2017).

The intestines of $N$. cincticeps at 6 days padp were fixed in $4 \%$ paraformaldehyde in PBS for $2 \mathrm{~h}$, and then permeabilized in $0.2 \%$ Triton-X for $1 \mathrm{~h}$. The intestines were then immunostained with antibodies of gelsolin-FITC or Pns11-rhodamine, as described previously (Chen et al. 2017). The resulting samples were observed using a Leica TCS SP5 inverted confocal microscope.

\section{RT-qPCR detection}

Total RNA of cultured cells or insects were extracted using TRIzol Reagent (Thermo Fisher Scientific). The first-strand cDNA of Pns11 and major outer capsid protein P8 of RGDV were synthesized by specific primers. cDNA of gelsolin or glyceraldehyde-3-phosphate dehydrogenase (GAPDH) was synthesized by oligo dT18 in the reaction mixture containing M-MLV Reverse Transcriptase (Promega, USA). The quantitative PCR assays were performed in Mastercycler realplex4 real-time PCR system (Eppendorf) using SYBR Green PCR Master Mix kit (Promega, USA). The relative abundance of the RGDV P8, Pns11, and gelsolin genes was normalized using GAPDH as the reference gene for each qPCR assay. The relative level of gene expression was analyzed by the $2^{-\Delta \Delta \mathrm{Ct}}$ (cycle threshold) method. The expression of P8, Pns11, and gelsolin in RGDV-infected cells at 12 hpi was normalized as 1.

For the quantification of the virus in insects, the concentration of the plasmid DNA including the RGDV P8 gene was determined using a NanoDrop 1000 (Thermo Fisher Scientific). The copy number of the plasmid including the RGDV P8 gene was calculated using the following formula: (amount $\times 6.022 \times 10^{23}$ )/(length $\times 1 \times$ $10^{9} \times 650$ ). A 10 -fold dilution series was prepared in RNase-free water to establish a standard curve. The cycle thresholds value was mapped to the standard curve 
of RGDV P8, and an equation as $y=-2.564 x+30.47$ $(\mathrm{y}=$ the logarithm of plasmid copy number to base $10, x=C t$ value, and $\left.R^{2}=0.9995\right)$ was generated. The viral genome copy as the log of the copy number per microgram of insect RNA was based on the equation above.

\section{dsRNAs synthesis in vitro}

Specific primers (forward primer 5 '-ATGCCCGACGA TGCCAGCGG-3', and reverse primer $5^{\prime}$-GTCAGAGG GACAGTCCTTGC-3') with a T7 RNA polymerase promoter $\left(5^{\prime}\right.$-TAATACGACTCACTATAGGG-3') at the $5^{\prime}$ terminal end were designed to amplify a region of approximately $540 \mathrm{bp}$ of the gelsolin gene. The full length of the GFP gene sequence with the T7 RNA polymerase promoter at the $5^{\prime}$ terminus was also amplified. Then, PCR products were synthesized into dsRNAs in vitro using the T7 RiboMAX (TM) Express RNAi System (Promega) according to the manufacturer's protocol. These dsRNAs were purified and examined for integrity using agarose gel electrophoresis; the quantity was determined using a NanoDrop 1000 (Thermo Fisher Scientific).

\section{Delivery of synthesized dsRNAs to cultured cells and insect bodies}

To transfect cultured cells of $N$. cincticeps, $16 \mu \mathrm{g}$ dsRNAs and $12 \mu \mathrm{L}$ cellfectin II Reagent (Thermo Fisher Scientific) were separately mixed with $100 \mu \mathrm{L}$ complete medium without fetal bovine serum and antibiotics (Kimura and Omura 1988). Then, these two solutions were gently mixed together and incubated for $20 \mathrm{~min}$ at room temperature, as described previously (Chen et al. 2017). Thereafter, cultured cells were successively incubated with the mixture for $8 \mathrm{~h}$ and inoculated with purified RGDV at a MOI of 1 for $2 \mathrm{~h}$. The cultured cells were recovered for complete culture.

Nonviruliferous second-instar nymphs were allowed to feed on plants infected with RGDV for 3 days, then microinjected with dsRNA (about $0.05 \mu \mathrm{g} /$ insect) at the intersegmental region of the thorax using a Nanoject II Auto-Nanoliter Injector (Spring), as described previously (Chen et al. 2015b). The insects were collected 6 days padp for testing.

\section{Viruliferous rate of leafhoppers treated with dsRNAs}

One hundred nonviruliferous second-instar nymphs were microinjected with dsRNAs (dsgeloslin or dsGFP) (approximately $0.05 \mu \mathrm{g} /$ insect). These nymphs were allowed to feed on plants infected with RGDV for 2 days. Thereafter, these dsRNA-treated insects were allowed to feed on healthy rice seedlings. At 4 days padp, the total RNA of insects was individually extracted to examine the presence of transcripts for the RGDV P8 gene. The viruliferous rate was determined based on the number of positive insects/total number of insects.

\section{Statistics of insect survival}

One hundred second-instar nymphs of leafhoppers microinjected with dsgelsolin or dsGFP (about $0.05 \mu \mathrm{g} /$ insect) were individually reared on a healthy rice seedlings in a single glass tube for 15 days. The number of living insects was counted each day. The mortality rate was determined based on the number of living insects/total number of insects.

\section{Statistical analyses}

All data for cultured cells and insects were analyzed with a one- or two-tailed t-test in GraphPad Prism 6. Data were back-transformed after analysis for presentation in the text and figures.

\section{Additional file}

Additional file 1: Figure S1. The effect of the knockdown of gelsolin on the survival rate of insects. Nonviruliferous second-instar nymphs were microinjected with dsRNAs and monitored daily for survival over 15 days. (TIF 6054 kb)

\section{Abbreviations \\ ABTM: Actin-based tubule motility; dsgelsolin: dsRNAs specific for gelsolin; dsGFP: dsRNAs specific for green fluorescent protein; dsRNA: Double- stranded RNA; FITC: Fluorescein-5-isothiocyanate; GAPDH: Glyceraldehyde-3- phosphate dehydrogenase; GFP: Green fluorescent protein; HBV: Hepatitis B virus; HIV-1: Human immunodeficiency virus type 1; IPTG: Isopropyl- $\beta-D-$ thiogalactopyranoside; MOI: Multiplicity of infection; MVM: Minute virus of mice; padp: Post-first access to diseased plants; PBS: Phosphate buffered saline; RDV: Rice dwarf virus; RGDV: Rice gall dwarf virus; RNAi: RNA interference; RSV: Rice stripe virus; SRBSDV: Southern rice black-streaked dwarf} virus; Tmod: Tropomodulin; $\mathrm{Y} 2 \mathrm{H}$ : Yeast two-hybrid assay

\section{Acknowledgements}

We thank Dr. Toshihiro Omura (National Agricultural Research Center, Japan) for providing the antibodies against Pns11 of RGDV.

\section{Funding}

This study was supported by the National Natural Science Foundation of China under grant numbers 31730071 and 31772124, the Natural Science Foundation of Fujian Province, China under grant number 2017 J06011, the National Key R\&D Program of China under grant number 2017YFD0200900, and the Program for New Century Excellent Talents in Fujian Province University under grant number Kla18057A.

\section{Availability of data and materials}

The datasets used and/or analyzed during the current study are available from the corresponding author on reasonable request.

\section{Authors' contributions}

QC and LZ designed the research and QC and TW wrote the manuscript. QC, $L Z, P Z, D J, Y L$ and $Q M$ performed the research and analyzed and interpreted the data. All authors read and approved the final manuscript.

Ethics approval and consent to participate Not applicable.

Consent for publication

Not applicable. 


\section{Competing interests}

The authors declare that they have no competing interests.

\section{Author details}

${ }^{1}$ Fujian Province Key Laboratory of Plant Virology, Institute of Plant Virology, State Key Laboratory of Ecological Pest Control for Fujian and Taiwan Crops, Vector-borne Virus Research Center, Fujian Agriculture and Forestry University, Fuzhou, Fujian 350002, People's Republic of China. ${ }^{2}$ Key Laboratory of Pest Management of Horticultural Crop of Hunan Province, Hunan Plant Protection Institute, Hunan Academy of Agricultural Science, Changsha 410125, People's Republic of China.

Received: 15 March 2019 Accepted: 6 May 2019

Published online: 23 May 2019

\section{References}

Attoui H, Mertens PPC, Becnel J, Belaganahalli S, Bergoin M, Brussaard CP, et al. Family Reoviridae. In: King AMQ, Adams MJ, Carstens EB, Lefkowitz EJ, editors. Virus taxonomy: ninth report of the international committee for the taxonomy of viruses. London: Elsevier Academic Press; 2012. p. 546-639.

Bar S, Daeffler L, Rommelaere J, Nuesch JP. Vesicular egress of non-enveloped lytic parvoviruses depends on gelsolin functioning. PLoS Pathog. 2008;4: e1000126.

Chen $\mathrm{H}$, Zheng $\mathrm{L}$, Jia $\mathrm{D}$, Zhang $\mathrm{P}$, Chen $\mathrm{Q}$, Liu $\mathrm{Q}$, et al. Rice gall dwarf virus exploits tubules to facilitate viral spread among cultured insect vector cells derived from leafhopper Recilia dorsalis. Front Microbiol. 2013;4:206.

Chen Q, Chen H, Mao Q, Liu Q, Shimizu T, Uehara-Ichiki T, et al. Tubular structure induced by a plant virus facilitates viral spread in its vector insect. PLoS Pathog. 2012;8:e1003032.

Chen $\mathrm{Q}$, Wang H, Ren T, Xie L, Wei T. Interaction between non-structural protein Pns10 of Rice dwarf virus and cytoplasmic actin of leafhoppers is correlated with insect vector specificity. J Gen Virol. 2015a;96:933-8.

Chen Q, Wei T. Viral receptors of the gut: insect-borne propagative plant viruses of agricultural importance. Curr Opin Insect Sci. 2016;16:9-13.

Chen Q, Zhang L, Chen H, Xie L, Wei T. Nonstructural protein Pns4 of Rice dwarf virus is essential for viral infection in its insect vector. Virol J. 2015b;12:211.

Chen Q, Zhang L, Zhang Y, Mao Q, Wei T. Tubules of plant reoviruses exploit tropomodulin to regulate actin-based tubule motility in insect vector. Sci Rep. 2017;7:38563.

Cheng G, Liu Y, Wang P, Xiao X. Mosquito defense strategies against viral infection. Trends Parasitol. 2016:32:177-86.

Coluccio LM. An end in sight: tropomodulin. J Cell Biol. 1994;127:1497-9.

Cooper JA, Schafer DA. Control of actin assembly and disassembly at filament ends. Curr Opin Cell Biol. 2000;12:97-103.

Dietzgen RG, Mann KS, Johnson KN. Plant virus-insect vector interactions: current and potential future research directions. Viruses. 2016;8:303.

Garcia-Exposito L, Ziglio S, Barroso-Gonzalez J, de Armas-Rillo L, Valera MS, Zipeto $D$, et al. Gelsolin activity controls efficient early HIV-1 infection. Retrovirology. 2013;10:39.

Jia D, Chen H, Zheng A, Chen Q, Liu Q, Xie L, et al. Development of an insect vector cell culture and RNA interference system to investigate the functional role of fijivirus replication protein. J Virol. 2012;86:5800-7.

Jia DS, Mao QZ, Chen HY, Wang AM, Liu YY, Wang HT, et al. Virus-induced tubule: a vehicle for rapid spread of virions through basal lamina from midgut epithelium in the insect vector. J Virol. 2014;88:10488-500.

Kimura I, Omura T. Leafhopper cell cultures as a means for Phytoreovirus research. Adv Dis Vector Res. 1988;5:111-35.

Kwiatkowski DJ, Stossel TP, Orkin SH, Mole JE, Colten HR, Yin HL. Plasma and cytoplasmic gelsolins are encoded by a single gene and contain a duplicated actin-binding domain. Nature. 1986;323:455-8.

Lan $H$, Wang $H$, Chen $Q$, Chen $H$, Jia D, Mao Q, et al. Small interfering RNA pathway modulates persistent infection of a plant virus in its insect vector. Sci Rep. 2016;6:20699.

Liao Z, Mao Q, Li J, Lu C, Wu W, Chen H, et al. Virus-induced tubules: a vehicle for spread of virions into ovary oocyte cells of an insect vector. Front Microbiol. 2017;8:475.

Liu W, Gray S, Huo Y, Li L, Wei T, Wang X. Proteomic analysis of interaction between a plant virus and its vector insect reveals new functions of hemipteran cuticular protein. Mol Cell Proteomics. 2015;14:2229-42.
Liu Y, Jia D, Chen H, Chen Q, Xie L, Wu Z, et al. The P7-1 protein of southern rice black-streaked dwarf virus, a fijivirus, induces the formation of tubular structures in insect cells. Arch Virol. 2011;156:1729-36.

Lu M, Witke W, Kwiatkowski DJ, Kosik KS. Delayed retraction of filopodia in gelsolin null mice. J Cell Biol. 1997;138:1279-87.

Mao Q, Liao Z, Li J, Liu Y, Wu W, Chen H, et al. Filamentous structures induced by a phytoreovirus mediate viral release from salivary glands in its insect vector. J Virol. 2017;91:e00265-17.

Mar T, Liu W, Wang X. Proteomic analysis of interaction between P7-1 of southern rice black-streaked dwarf virus and the insect vector reveals diverse insect proteins involved in successful transmission. J Proteome. 2014;102:83-97.

Marrocco C, Rinalducci S, Mohamadkhani A, D'Amici GM, Zolla L. Plasma gelsolin protein: a candidate biomarker for hepatitis B-associated liver cirrhosis identified by proteomic approach. Blood Transfus. 2010;8(Suppl 3):s105-12.

Mazur AJ, Gremm D, Dansranjavin T, Litwin M, Jockusch BM, Wegner A, et al. Modulation of actin filament dynamics by actin-binding proteins residing in lamellipodia. Eur J Cell Biol. 2010;89:402-13.

Moriyasu Y, Ishikawa K, Kikuchi A, Imanishi S, Tomita S, Akutsu K, et al. Sequence analysis of Pns11, a nonstructural protein of Rice gall dwarf virus, and its expression and detection in infected rice plants and vector insects. Virus Genes. 2000;20:237-41.

Moriyasu Y, Maruyama-Funatsuki W, Kikuchi A, Ichimi K, Zhong B, Yan J, et al. Molecular analysis of the genome segments S1, S4, S6, S7 and S12 of a Rice gall dwarf virus isolate from Thailand; completion of the genomic sequence. Arch Virol. 2007;152:1315-22.

Mudry RE, Perry CN, Richards M, Fowler VM, Gregorio CC. The interaction of tropomodulin with tropomyosin stabilizes thin filaments in cardiac myocytes. J Cell Biol. 2003;162:1057-68.

Peddada N, Sagar A, Rathore YS, Choudhary V, Pattnaik UB, Khatri N, et al. Global shapes of F-actin depolymerization-competent minimal gelsolins: insight into the role of $\mathrm{g} 2-\mathrm{g} 3$ linker in $\mathrm{pH} / \mathrm{Ca}^{2+}$ insensitivity of the first half. J Biol Chem. 2013;288:28266-82.

Safiejko-Mroczka B, Bell PB Jr. Reorganization of the actin cytoskeleton in the protruding lamellae of human fibroblasts. Cell Motil Cytoskeleton. 2001;50: $13-32$.

Tang WC, Lin RJ, Liao CL, Lin YL. Rab18 facilitates dengue virus infection by targeting fatty acid synthase to sites of viral replication. J Virol. 2014;88:6793-804.

Vaidyanathan R, Scott TW. Apoptosis in mosquito midgut epithelia associated with West Nile virus infection. Apoptosis. 2006;11:1643-51.

Wang H, Gort T, Boyle DL, Clem RJ. Effects of manipulating apoptosis on Sindbis virus infection of Aedes aegypti mosquitoes. J Virol. 2012;86:6546-54.

Wei T, Kikuchi A, Moriyasu Y, Suzuki N, Shimizu T, Hagiwara K, et al. The spread of Rice dwarf virus among cells of its insect vector exploits virus-induced tubular structures. J Virol. 2006:80:8593-602.

Wei T, Li Y. Rice reoviruses in insect vectors. Annu Rev Phytopathol. 2016;54:99120

Wei T, Miyazaki N, Uehara-Ichiki T, Hibino H, Shimizu T, Netsu O, et al. Threedimensional analysis of the association of viral particles with mitochondria during the replication of Rice gall dwarf virus. J Mol Biol. 2011;410:436-46.

Wei T, Shimizu T, Omura T. Endomembranes and myosin mediate assembly into tubules of Pns10 of Rice dwarf virus and intercellular spreading of the virus in cultured insect vector cells. Virology. 2008;372:349-56.

Xi Z, Ramirez JL, Dimopoulos G. The Aedes aegypti toll pathway controls dengue virus infection. PLoS Pathog. 2008;4:e1000098.

Zhang HM, Xin X, Yang J, Chen JP, Wang JM, Adams MJ. Completion of the sequence of Rice gall dwarf virus from Guangxi, China. Arch Virol. 2008;153: 1737-41.

Zheng $L$, Chen $H$, Liu H, Xie L, Wei T. Assembly of viroplasms by viral nonstructural protein Pns9 is essential for persistent infection of Rice gall dwarf virus in its insect vector. Virus Res. 2015;196:162-9. 